\title{
A comparative morphological study of the ligamen- tum lamina nuchae and ligamenta interspinalia reveals enthesis patterns at the sites of attachment from the second to seventh cervical vertebrae in four extant species of Equus
}

\section{Sharon May-Davis*, Robert Hunter, Wendy Y. Brown}

University of New England, Canine and Equine Research Group, Armidale, New South Wales, Australia maydavis@bigpond.com; anthropal@outlook.com; wbrown@une.edu.au

\begin{abstract}
Morphological variation in the attachment sites of the ligamentum lamina nuchae and ligamenta interspinalia to the 2nd to 7th cervical vertebrae is described in four species of Equus, and enthesis patterns in relation to these attachment sites are explored. This comparative study provides new insights with respect to this variation in the zebrine and asinine clades within Equus. In zebrines (Equus caballus, E. quagga boehmi and E. przewalskii), the ligamentum lamina nuchae attaches to the eminence of the cervical dorsal spines from either the 2 nd to 5 th, and or 2 nd to 7 th. These attachments resemble digits and between each digit an aperture is apparent. The ligamenta interspinalia attach to the base of each cervical dorsal spine from the $2 \mathrm{nd}$ to 7 th before progressing caudally onto the thoracic dorsal spines. Three enthesis patterns per cervical dorsal spine correspond to the size and shape of these ligaments. In contrast, in asinines (as represented by Equus asinus), the ligamentum lamina nuchae attaches without digitation to the complete sagittal ridge of the cervical vertebra including the dorsal spine and exhibits no ligamenta interspinalia. Consequently, the enthesis pattern is represented by a raised sagittal ridge only, excluding the 7 th cervical dorsal spine. These differences between the ligamentum lamina nuchae and ligamenta interspinalia attachment sites create distinct enthesis patterns that differentiate zebrines from asinines. Findings include: differing ligamentous morphology; associated enthesis patterns corresponding to attachment sites of the ligaments to the cervical vertebrae; influences in dorsal spine morphology subject to the presence or absence of the ligaments; and the postulation of evolutionary functional adaptions in response to environmental influences between the two Equus clades. These results may be useful for palaeontologists in the identification of isolated cervical vertebrae.
\end{abstract}

\section{INTRODUCTION}

Comparative morphology examines the body structures of different species, partly to understand the adaptive changes they have undergone through evolutionary processes (Blits 1999). The fossil record and comparative morphology of American hippomorphs, beginning with ancestral Eohippus some 55-60 million years ago until the present day, where only one extant genus, Equus, exists (MacFadden 1992; Orlando 2015), have been studied extensively. Extant Equus comprises seven species in two clades - the zebrines (zebras and horses) and the asinine (asses, donkeys, kiangs, kulans, onagers) (MacFadden 1992, 2005; Vilstrup et al. 2013). The two clades are distinguished by distinct phenotypic variations of the splanchnologia, angiologia, integument, and musculoskeletal system (MacFadden 1992, 2005; Burnham 2002; Orlando 2015; Burden and Thiemann 2015). This became further evident when a recent comparative study investigated the cervical attachments of the lig. lamina nuchae (the sheet portion of the 2-part ligamentum nuchae) in four species of Equus: E. caballus, E. przewalskii, E. asinus and E. quagga boehmi (May-Davis et al. 2018). 
May-Davis et al. - Comparative morphology of ligaments in Equus

The findings revealed the lig. lamina nuchae in E. przewalskii, E. asinus and E. quagga boehmi attaches from the 2nd -7 th cervical vertebrae, whereas in E. caballus the most common attachment is from the 2nd-5th (MayDavis and Kleine 2014; May-Davis et al. 2018, 2020). Previous human studies relating to the attachment sites of the lig. nuchae have focused on the strong elastic cord-like portion referred to as the ligamentum funiculus nuchae (Benjamin et al. 2006; Schlecht 2012; Apostolakos et al. 2014). This ligament has also been studied in E. caballus where the emphasis centred on enthesis patterns and enthesopathies at the site of attachment to the external occipital protuberance (Bendrey 2008; Dyson 2011). Entheses are important sites of osteological modification associated with the insertions of tendons, ligaments, and joint capsules. Fundamentally, they are adaptations at the connective interface between soft and hard tissue structures that modify according to biomechanical load (Shaw and Benjamin 2007). When the load and directional forces increase, the osteological interface responds through modifications in size, shape and pattern to provide a more secure and stable anchor for soft tissue structures to effectively transmit their forces (Frost 1994; Benjamin et al. 2006; Shaw and Benjamin 2007). These modifications leave enthesis patterns on the bone that can be mapped and read post-mortem, even in archaeological specimens (Sanchez et al. 2013). As this adaptation applies to the occipital attachment of the lig. funiculus nuchae, the same principle should apply to the cervical attachments of the lig. lamina nuchae.

Therefore, the aim of this study is to identify through a comparative gross anatomical study the insertion points of the lig. lamina nuchae and the anatomically contiguous ligg. interspinalia onto the cervical vertebrae from the 2 nd to the 7th in E. caballus, E. przewalskii, E. quagga boehmi and E. asinus. In addition, examine the osteological changes (entheses) at the sites of attachment with the view to provide insights into the functional evolutionary history associated with the variations between the two clades.

\section{METHODS}

Ethical statement: No equids were euthanised for the purpose of this study and all observations were obtained postmortem.

Anatomic terminology: The anatomic terms for this manuscript followed the terminology from Nomina Anatomica Veterinaria 6th edition (Gasse et al. 2017).

Functional anatomy: In E. przewalskii, E. quagga boehmi and E. asinus, the lig. lamina nuchae consists of a bilateral sheet of yellow ligamentous elastin fibres that arise from the dorsal spines of the 2 nd and 3 rd thoracic vertebrae and lig. funiculus nuchae; after which they traverse cranioventral and insert onto the dorsal spines of the $2 \mathrm{nd}$ to 7 th cervical vertebrae. In E. caballus, the insertions normally attach from 2nd - 5th (May-Davis and Kleine 2014), but variations include insertions that extend to the 6th and/or 7th cervical vertebra (May-Davis et al. 2018, 2020). Each insertion is known as a digitation due to the finger like projections of attachment (Sisson 1975; May-Davis and Kleine 2014; May-Davis et al. 2018). The lig. lamina nuchae functions to support and stabilize the cervical vertebrae while assisting locomotion (Gellman and Bertram 2002; Dunbar et al. 2008).

The cervical ligg. interspinalia comprise a bilateral pair of yellow elastin ligament that extend longitudinally between the dorsal spines from the 2 nd cervical vertebra through to the 1 st thoracic vertebra. They function to assist in the stabilization of the cervical vertebrae during movement; with a special emphasis for when the cervical spine is in flexion (McFadyean 1884; Sisson 1975).

Animal details and dissection: In this study, one mature animal represented each of the four species: Equus caballus ( 6 year old female), E. asinus (15 year old male), E. przewalskii (11 year old male) and E. quagga boehmi (23 year old female).

Equus caballus and E. asinus were sourced from an abattoir in Australia; E. przewalskii from Hortobagy Nature Reserve in Hungary and E. quagga boehmi from Emmen Zoo in The Netherlands.

The dissections revealing the attachments of the lig. lamina nuchae to the cervical vertebrae followed the protocol established by May-Davis et al. (2018). Gross comparative examination of the lig. lamina nuchae and ligg. interspinalia attachment sites were recorded prior to preparation for comparative enthesis examination.

Maceration and degreasing: Upon completion of the dissection and examination of the lig. lamina nuchae and the ligg. interspinalia sites of attachment in all four specimens, the cervical vertebrae were disarticulated and prepared for maceration.

Preparation of the cervical vertebrae followed the protocols established by Hangey and Dingly (1985) in skeletal preparation, maceration by cooking and degreasing, with several modifications. The water was not boiled and cooking utensils of metal other than stainless steel were used. A simmering temperature of $80^{\circ} \mathrm{C}$ or slightly below is preferable with the contents in the pot included. The contents were removed from the heat after four to eight hours (dependent on vertebral size). The process was repeated if fleshy remains interfered with identification of the enthesis patterns.

The cleaned bones were submerged in a sodium bicarbonate solution (ratio - 85 grams $\mathrm{NaHCO}^{3}$ to 1 litre water). This process of maceration was repeated by fol- 
lowing the cooking protocol (above) and cooling process recommended by Hangey and Dingly (1985). Once the cervical vertebrae were cool to handle, they were rinsed in warm water $\left(-40^{\circ} \mathrm{C}\right)$ and placed on an open-air rack so the liquids could drain without pooling around the bones.

Identifying the entheses: When the specimens were completely dry, the enthesis patterns were identified by comparing the gross anatomical record of each attachment point from the lig. lamina nuchae and the ligg. interspinalia to their corresponding dorsal spines. The dorsal spines and enthesis patterns were then photographed in portrait and macro imagery.

Photography: Camera - Canon Rebel SL2 EOS 200D. (C) Canon Inc, 30-2 Shimonmaruko 3-chome, Ohta-ku, Tokyo 146-8501, Japan.

Lens $x 2-1$. Canon EFS $18-55 \mathrm{~mm}$ image stabilizer, $0.25 \mathrm{~m} / 0 / 8 \mathrm{ft}-\infty$. 2. Canon EF $100 \mathrm{~mm} \mathrm{f} / 2.8 \mathrm{~L}$ Macro IS USM. (C) Canon Inc, 30-2 Shimonmaruku 3-chome, Ohtaku, Tokyo 146-8501, Japan.

Tripod - Manfrotto 290 series with adjustable height and platform for camera angles. (C) Vitec Imaging Solutions Spa, via Valsugana, 100, 36022 Cassola (VI) Italy.

\section{RESULTS}

\section{Morphology of the Ligamentum lamina nuchae and Ligamenta interspinalia Zebrine - morphology of the ligamentum lamina} nuchae: The lig. lamina nuchae attaches to the central eminence of the dorsal spines of either C2-C5 (E. caballus) or C2-C7 (E. przewalskii and E. quagga boehmi). The fibres then pass dorso-caudally, attaching to the dorsal spines of the 2nd and 3rd thoracic vertebrae and lig. funiculus nuchae. The morphology of each cervical attachment is unique to the individual and each digitation unifies dorsally before blending into a lamina sheet (Fig. 1). The largest digitation attaches to the 2 nd cervical vertebra and each digitation decreases in size in each successive caudal vertebra. From the 2 nd -5 th cervical vertebrae, some fibres attach to the ligg. interspinalia (Fig. 1).

The location of the dorsal spines of the 3rd -7 th cervical vertebrae becomes more cranial along the sagittal plane and accordingly, the attachment points and digitations of the lig. lamina nuchae correspond with the anatomical positioning of the dorsal spines.

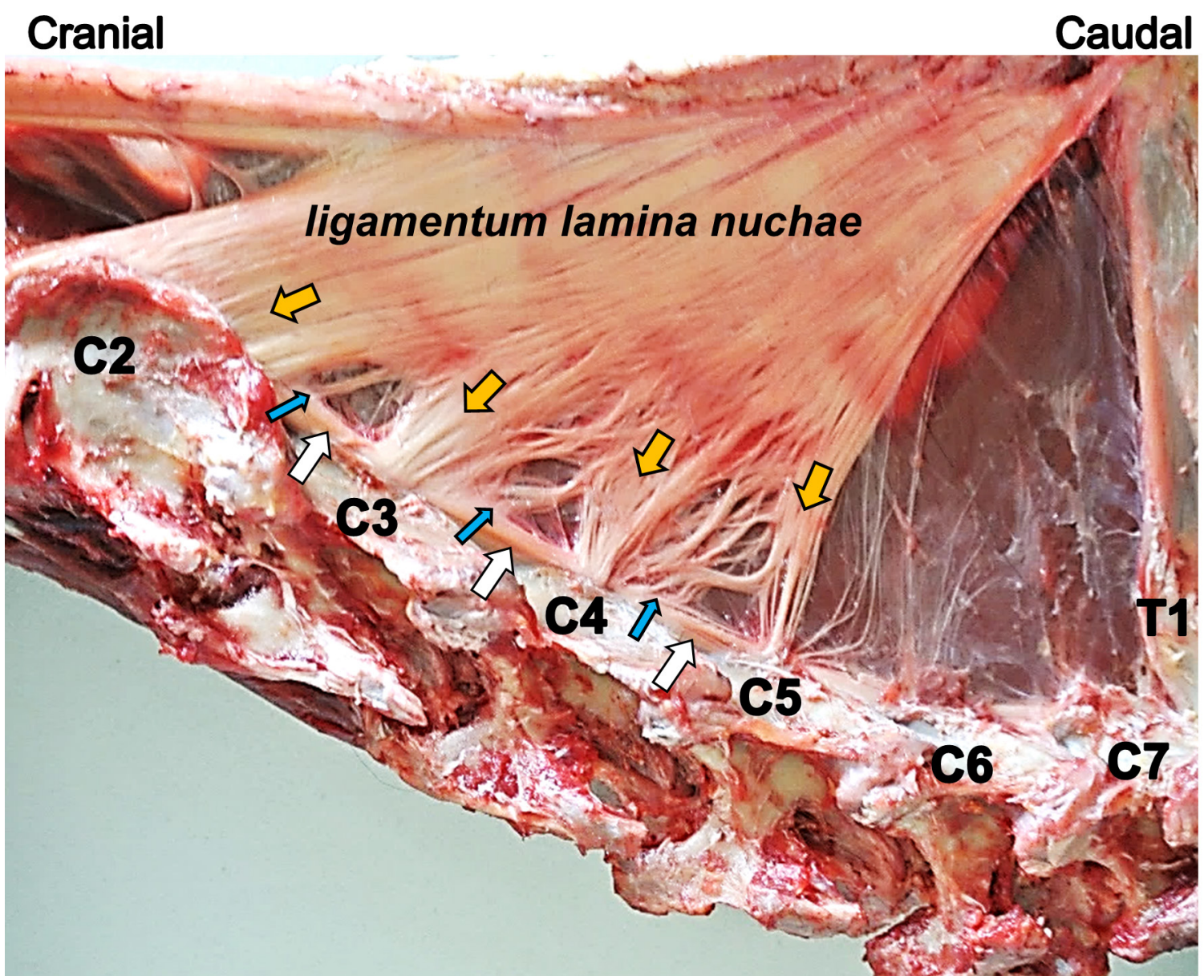

Figure 1. Ligamentum lamina nuchae with digitations (orange arrows) from the 2 nd -5 th cervical vertebrae in Equus caballus. Note: fibres from the ligamentum lamina nuchae attaching (blue arrows) to the ligamenta interspinalia (white arrows). 
May-Davis et al. - Comparative morphology of ligaments in Equus

Zebrine - morphology of the ligamentum lamina nuchae apertures: Triangular apertures form between digitations. Each aperture lies dorsal to its respective cervical articulation, creating sequential apertures from the 2nd cervical vertebra to the 1 st thoracic vertebra. Larger apertures are present in E. caballus and E. quagga boehmi than E. przewalskii. At the apex of each aperture, the lig. lamina nuchae fibres unite and form a continuous elastic lamina sheet (Figs. 1, 2).

\section{Zebrine - morphology of the ligamentum lamina nuchae associated with the 6 th and 7 th cervical} vertebrae: The morphology and number of fibres associated with the 6th and 7 th cervical vertebral digitations varied according to the individual. Equus caballus presented with none (Fig. 1); E. przewalskii presented with a more separated fibre morphology (Fig. 3A), while E. quagga boehmi presented a distinct cord-like morphology (Fig. 3B). In the presence of attachments from the 2 nd -7 th cervical vertebrae, the sequential triangular apertures elongated caudodorsal from the 5 th cervical vertebra to the 1 st thoracic vertebra (Fig. 3).

\section{Zebrine - morphology of the ligamenta interspin-}

alia: The ligaments attach to the base of the cranial margin of each vertebra (except the 2nd), caudal margin and sagittal ridge of each cervical dorsal spine except the 1st.
The diameter of the ligaments diminish in a cranio-caudal direction and fibre definition is more pronounced cranially than caudally. The distance between dorsal spines determines the length of the ligaments as it establishes the ventral base of each triangular aperture between the digitations of the lig. lamina nuchae (Figs. 1, 2, 4).

Where the lig. lamina nuchae attaches to its fibres for anchorage, it dorsally alters the morphology of the ligament, even to the point of separating fibres from the main body of the ligament (Fig. 4).

\section{Asinine - morphology of the ligamentum lam-} ina nuchae: The ligament attaches along the sagittal ridge from the cranial to caudal borders of the 2 nd to 7 th cervical vertebrae before traversing caudodorsally to the dorsal attachments (2nd and 3rd thoracic dorsal spines and lig. funiculus nuchae). The extensive number of fibres are larger in diameter from the 2 nd -4 th cervical vertebrae than the 5 th -7 th cervical vertebrae and appear tightly connected by fascia. There were no digitations or apertures as in zebrines (Fig. 5).

One significant morphological trait appears in the connection of the most caudal ligamentous fibre of the lig. lamina nuchae on each cervical vertebra. This fibre initially traversed caudally, bridging the articulation between the two cervical vertebrae before attaching to the cranial sagittal ridge of the contiguous caudal vertebra after which

\section{Caudal}

\section{Cranial}

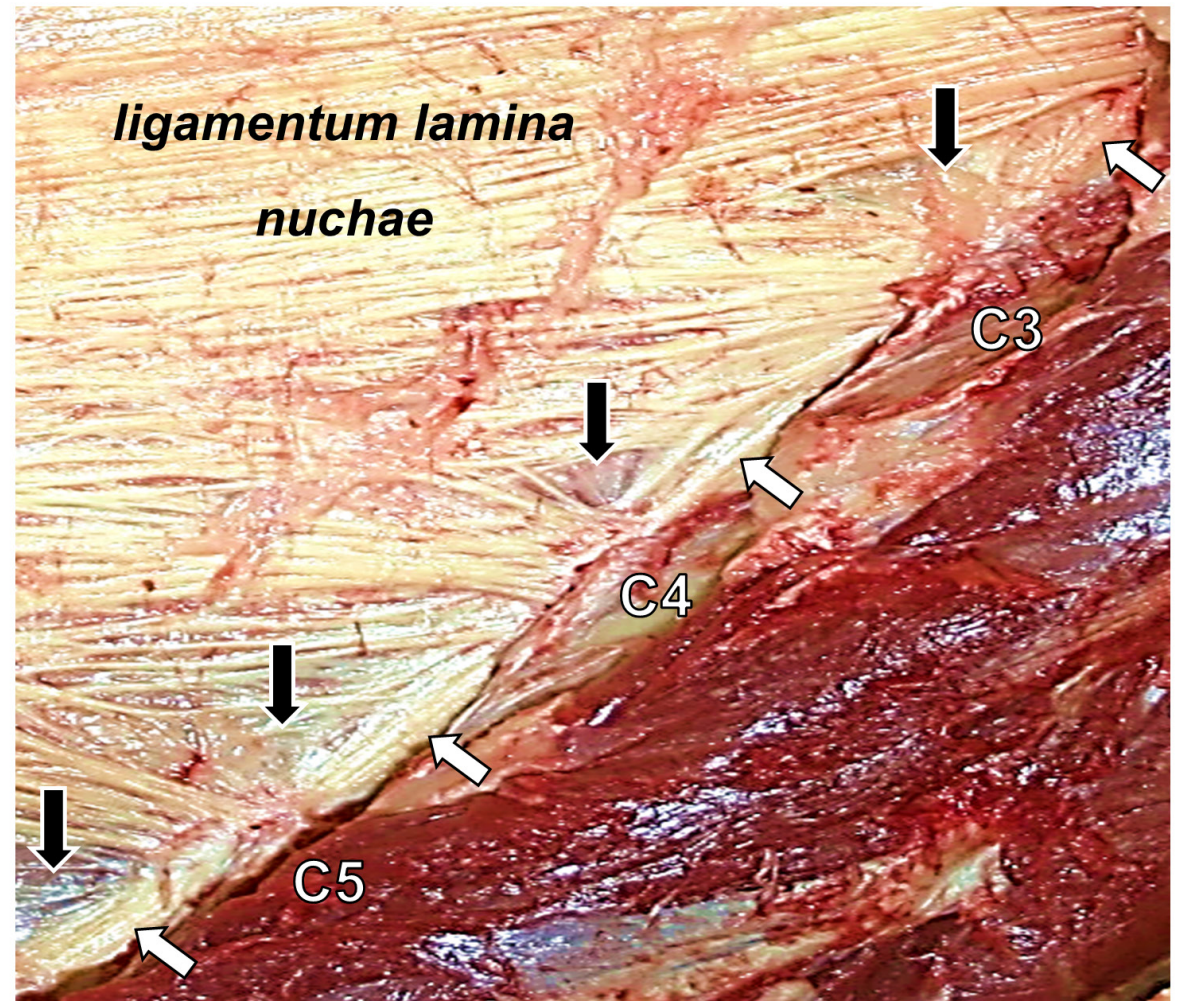

Figure 2. Sequential triangular apertures (black arrows) between the ligamentum lamina nuchae digitations and ligamenta interspinalia (white arrows) in Equus przewalskii. 

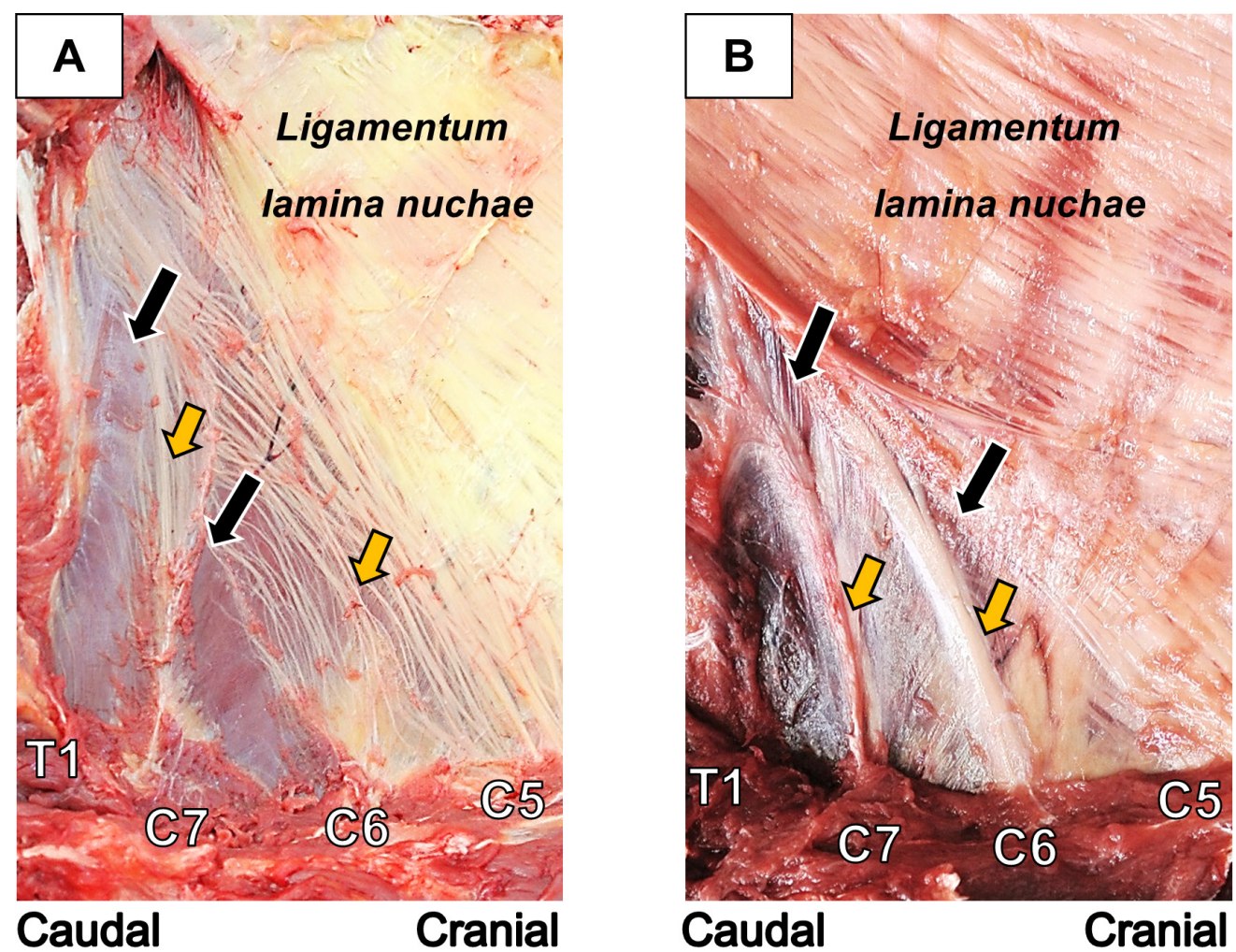

Caudal

Cranial

Figure 3. Variations of the ligamentum lamina nuchae digitations (orange arrows) from the 6 th and 7 th cervical vertebrae displaying elongated sequential triangular apertures (black arrows). A, Equus przewalskii with a separated morphology. B, Equus quagga boehmi with cord-like morphology.

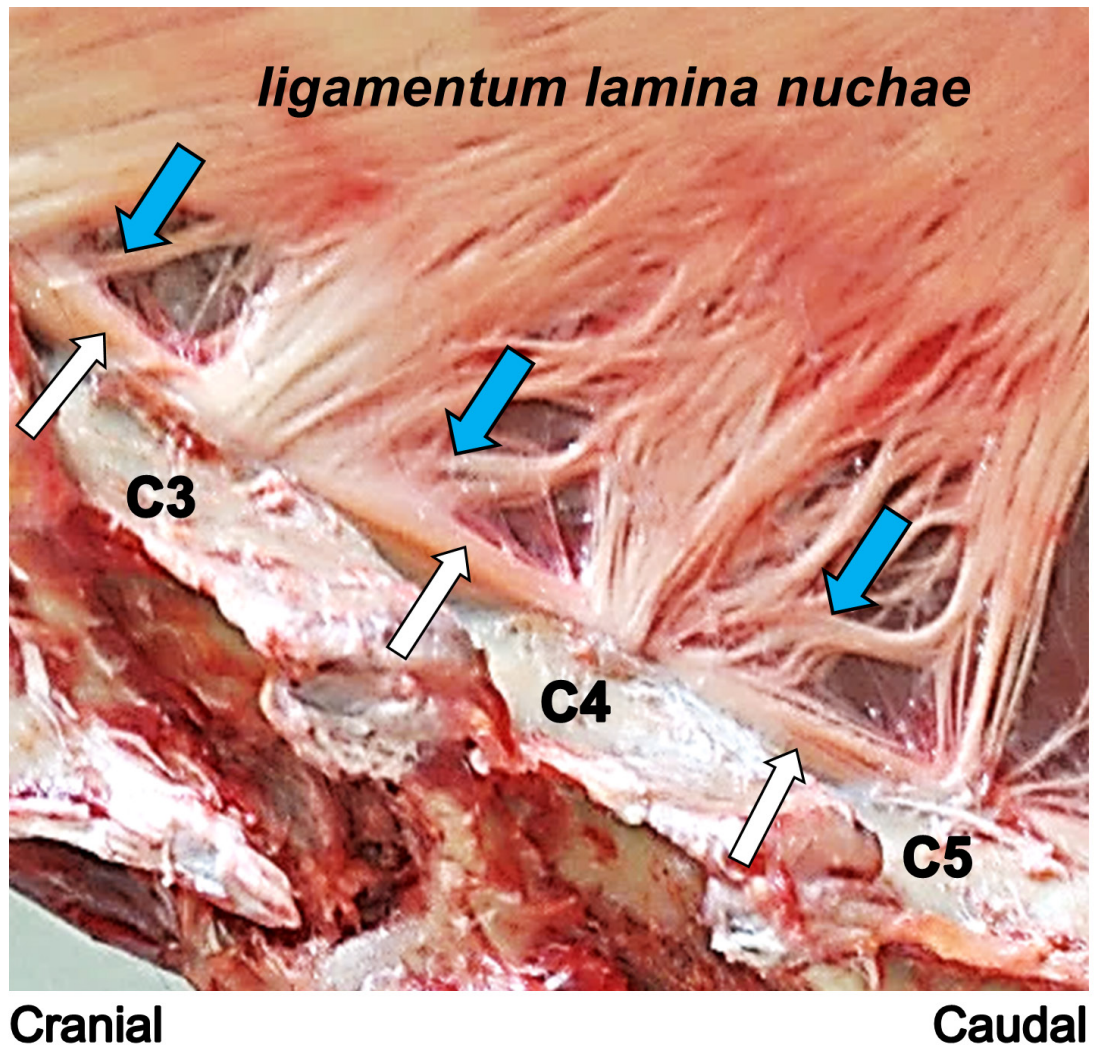

Figure 4. Ligamenta interspinalia (white arrows) noting the attachments of the ligamentum lamina nuchae digitations (blue arrows) from the 2 nd -5 th cervical vertebrae in Equus caballus. 


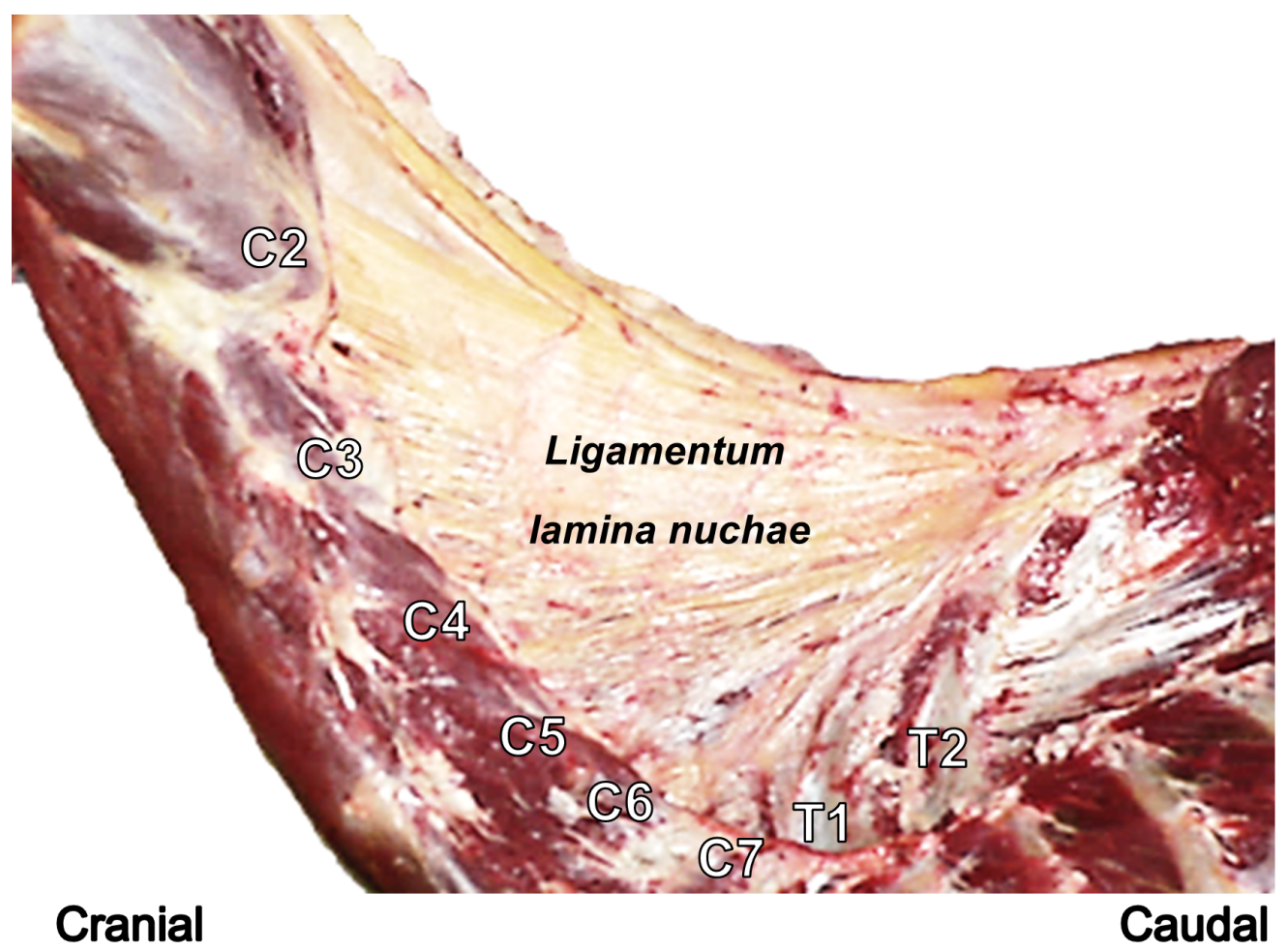

Figure 5. Ligamentum lamina nuchae from the 2nd - 7th cervical vertebrae in Equus asinus.

it traversed dorsally as in normal morphology. This in effect replaces the ligg. interspinalia, which appear to be absent (Fig. 6). Thereafter, the lig. lamina nuchae remains morphologically consistent with zebrines.
The Enthesis Patterns

Zebrine - enthesis patterns of the ligamentum lamina nuchae and ligamenta interspinalia: The morphology of the cervical dorsal spine reflects the size,

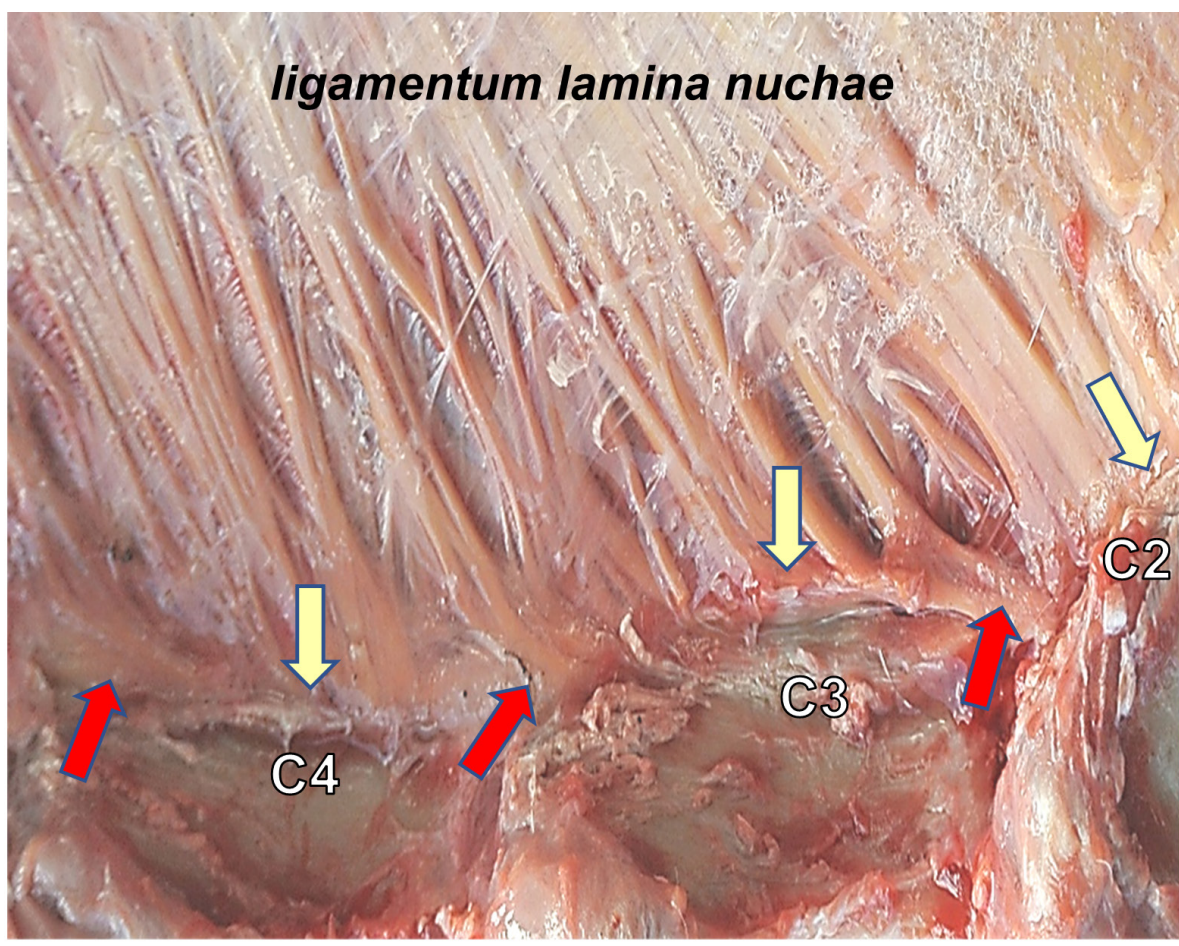

\section{Cranial}

Cranial

Figure 6. Attachment of the ligamentum lamina nuchae to the sagittal ridge of the cervical vertebrae (yellow arrows) with bridging fibres (red arrows) and no triangular apertures nor an apparent ligamenta interspinalia in Equus asinus. 
shape, and location of the ligamentous attachments (Fig. 7). Both anterior and posterior margins of each spine bear clear attachment areas; in addition, the anterior borders of the $3 \mathrm{rd}-7$ th cervical spines bear cranial extensions that trend larger cranio-caudally (Fig. 7B, C, D).

The enthesis patterns from the attachments of the lig. lamina nuchae and ligg. interspinalia exhibited by the 2nd 5 th cervical vertebrae remain relatively consistent between the three species (Fig. 7). The least consistent findings are associated with attachments of the lig. lamina nuchae to the 6th and 7th cervical vertebra, where variations of dorsal spine morphology and enthesis patterns were noted in direct correlation to the presence, size, and location of the attachments (Figs. 3, 7). Equus quagga boehmi presents the most distinct raised dorsal spine morphology and enthesis patterns relative to size, shape, and location when compared to E. przewalskii (Fig. 8). The variation in enthesis patterns between the 6th and 7 th cervical vertebrae in zebrines, relates to the presence, location, and size of the lig. lamina nuchae attachments (Figs. 7D, 8A, B).

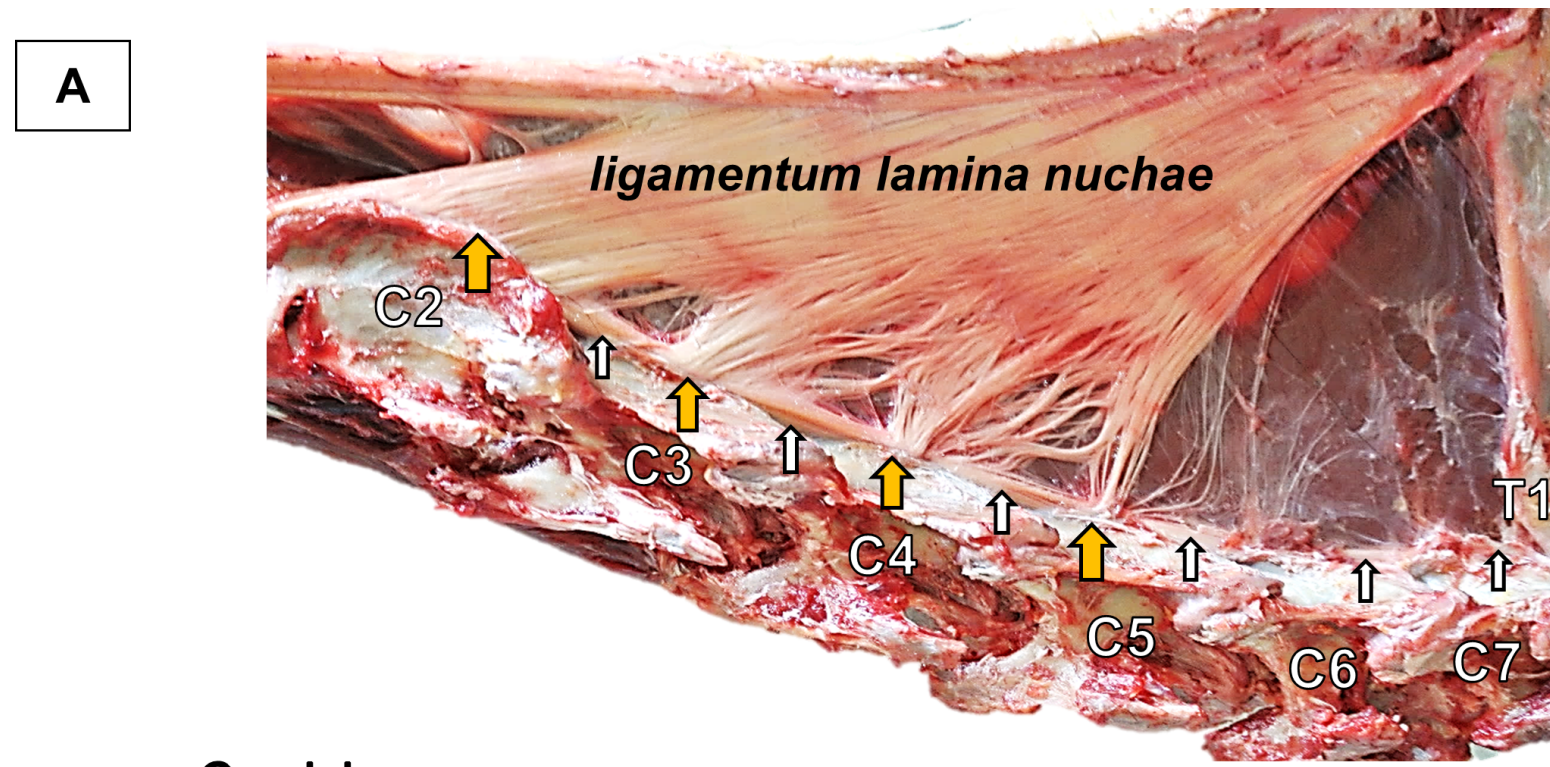

Cranial
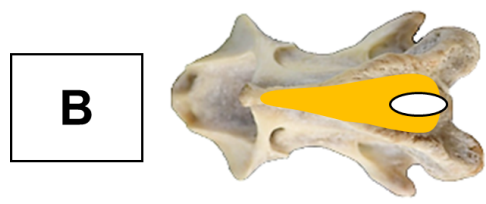

C2
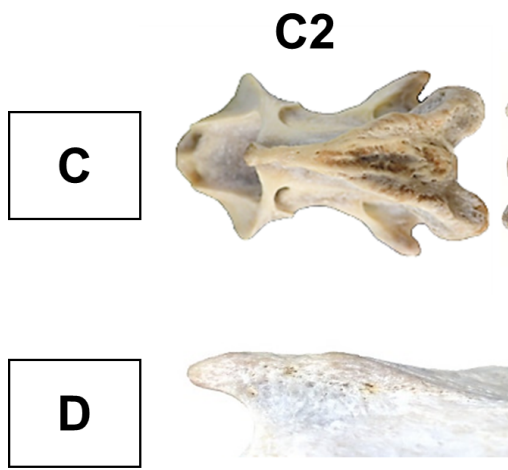

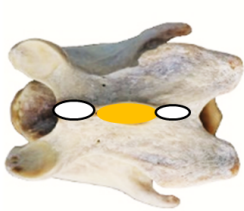

C3
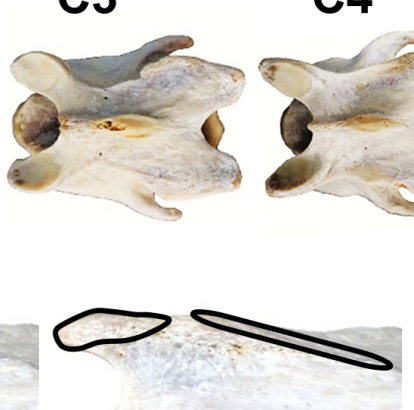

Dorsal spine C6

ligamentum lamina nuchae
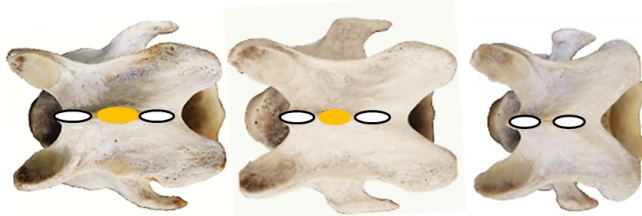

Caudal
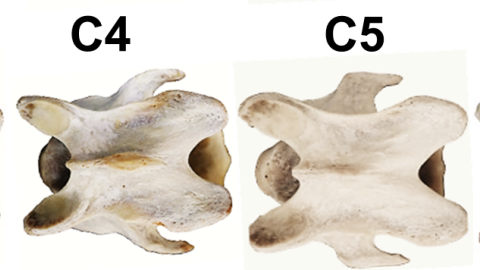

C6
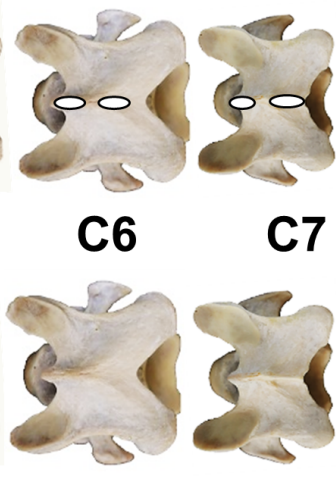

C7
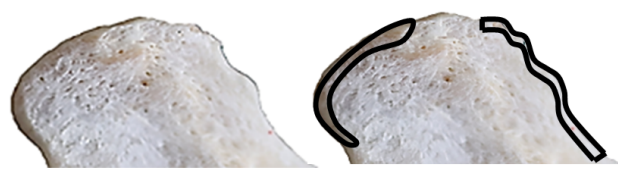

\section{Dorsal spine $\mathrm{C7}$}

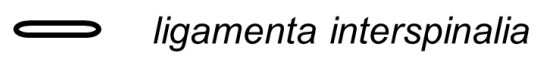

Figure 7. Attachment sites of the ligamentum lamina nuchae from the 2 nd -5 th cervical vertebrae and ligamenta interspinalia from the 2nd cervical vertebra to the 7 th cervical in E. caballus. A, the ligamentum lamina nuchae (orange arrows) and the ligamenta interspinalia (white arrows); $B$, dorsal view of the 2 nd -7 th cervical vertebrae showing corresponding mapped attachments sites; C, dorsal view of the enthesis patterns; D, lateral view of the 6th and 7th cervical dorsal spines noting mapped attachment sites of the ligamenta interspinalia. 


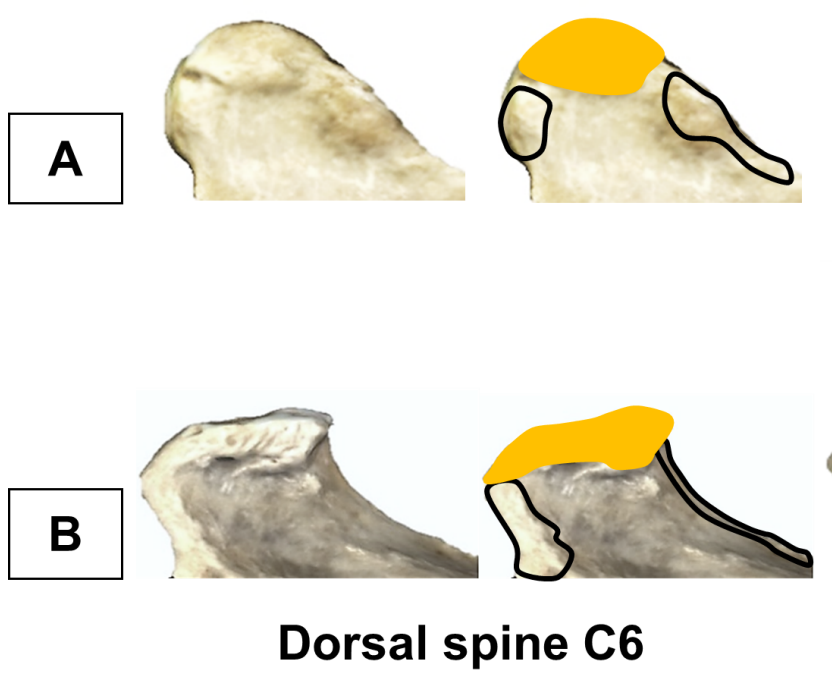

$\longrightarrow$ ligamentum lamina nuchae
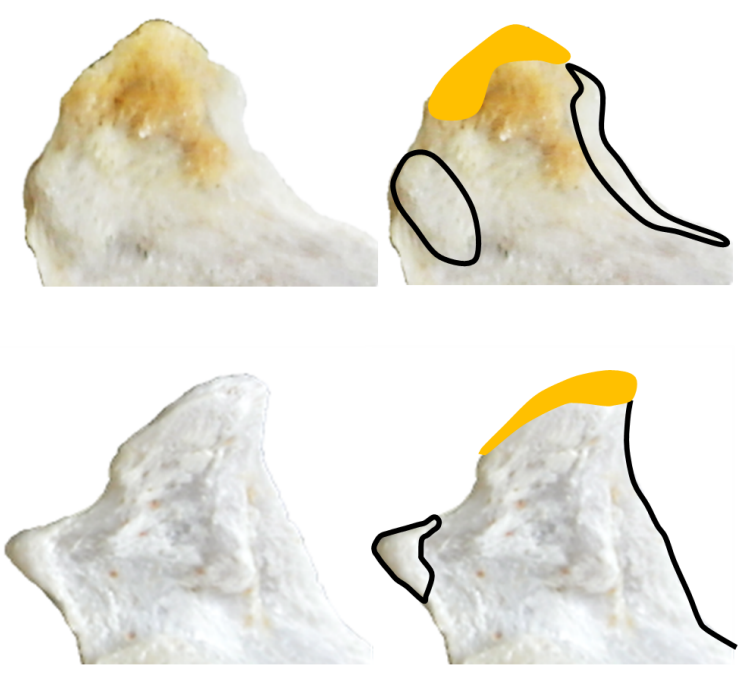

\section{Dorsal spine C7}

$\longrightarrow$ ligamenta interspinalia

Figure 8. Enthesis patterns of the ligamentum lamina nuchae and ligamenta interspinalia on the 6th and 7th cervical vertebrae. A, Equus przewalskii lateral view of the 6 th and 7 th cervical dorsal spines noting mapped attachment sites of the ligamentum lamina nuchae and ligamenta interspinalia. B, Equus quagga boehmi lateral view of the 6th and 7th cervical dorsal spines noting mapped attachment sites of the ligamentum lamina nuchae and ligamenta interspinalia.

When the lig. lamina nuchae is absent on the 6th cervical vertebra, the shape of the dorsal spine appears as a slightly raised elongation from the cranial to central aspect of the sagittal ridge. A prominent cranial extension is present with a lesser caudal modification noted on the dorsal spine, both of which are associated with the attachment sites of the ligg. interspinalia. This caudal enthesis pattern of the ligg. interspinalia is straight and elongated as it blends seamlessly into the sagittal ridge (Fig. 7D).

When the lig. lamina nuchae is absent from the 7th cervical vertebra, the eminence of the dorsal spine is flatter with no raised modifications indicative of an enthesis (Fig. 7D). When the lig. lamina nuchae is present on the 6th cervical vertebra, the attachment sites in E. przewalskii and E. quagga boehmi are predominantly central to the dorsal spine with enthesis patterns presenting chiefly as a focal modification indicative of the size of the digitation (Figs. 3, 8).

When the lig. lamina nuchae is present on the 7 th cervical vertebra, attachment sites are predominantly central to the dorsal spine with the enthesis patterns presenting chiefly as a focal modification with a modified apex (Fig. 8A, B). The shape of the dorsal spine may be an individual trait or age related, as the height bears no correlation to premortem specimen size. There are no specific correlative modifications associated with the cranial attachment sites of the ligg. interspinalia; however, the caudal entheses appear with a slight concavity prior to blending into the sagittal ridge (Fig. 8A, B), except in the vertebra depicted in Figure 7D, where there is no lig. lamina nuchae attachment involved
(Fig. 7A). It is important to emphasize that the enthesis patterns and modification of the dorsal spines correlates directly with the size of the lig. lamina nuchae.

Asinine - enthesis patterns of the ligamentum lamina nuchae: The lig. lamina nuchae extends from the $2 \mathrm{nd}-$ 7 th cervical vertebrae without digitations. It displays a wide cranial border at the sagittal ridge and tapers slightly towards the caudal border; this is reflected in the enthesis patterns. The width of the enthesis patterns on each cervical vertebra gradually decreases in size cranio-caudally (Fig. 9). The size of the enthesis patterns directly correlate to the location, size, and shape of the attachments. The 2 nd cervical vertebrae express the highest dorsal spine, while 7 th cervical vertebra is the second highest.

\section{Zebrine vs asinine - comparative morphology}

All equids examined in this study express lig. lamina nuchae attachments on respective cervical and thoracic dorsal spines and on the lig. funiculus nuchae. In zebrines, the ligament attaches to the dorsal spines of the cervical vertebrae with digitations and occasionally on the ligg. interspinalia, while the asinine expresses no digitations and the lig. lamina nuchae attaches entirely to the sagittal ridges of the vertebral spines. The ligg. interspinalia are present in all zebrines and exhibits similar morphology, but are absent in the asinine.

The 'curvate' (curved outward or convex) morphology of the 2nd cervical dorsal spine is the most consistent morphological feature among the equids in this study. Less consistent is the 'lunate' (semilunar or concave) 
morphology of the caudal margin of the 7 th cervical dorsal spine in the presence of the lig. lamina nuchae attachment. The dorsal spine of the 7th cervical vertebra in E. quagga boehmi (Fig. 8B) and E. asinus exhibits strong lig. lamina nuchae attachments (Fig. 9D) display similar 'falcate' (sickle/hooked shape) morphology; E. przewalskii also displays a strong attachment without the distinct 'falcate' morphology.

\section{DISCUSSION}

This study describes the comparative anatomy of the lig. lamina nuchae, ligg. interspinalia and corresponding enthesis patterns between four extant species of Equus representing both zebrine and asinine. The morphological findings demonstrated new anatomical insights, and variations between the two clades not previously reported, even though musculoskeletal similarities have been documented (Herman

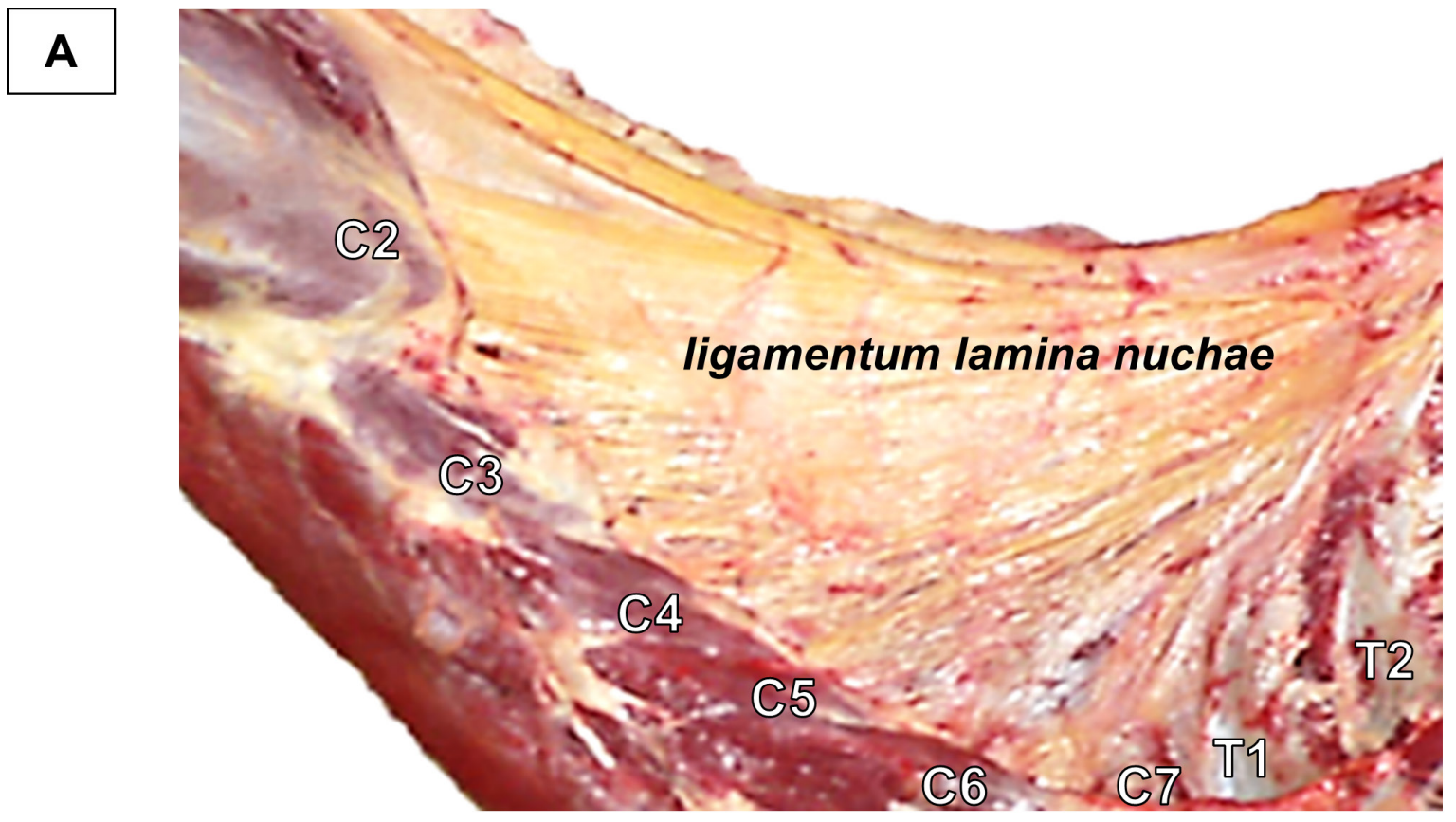

\section{Cranial}

B

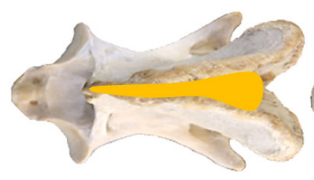

C2
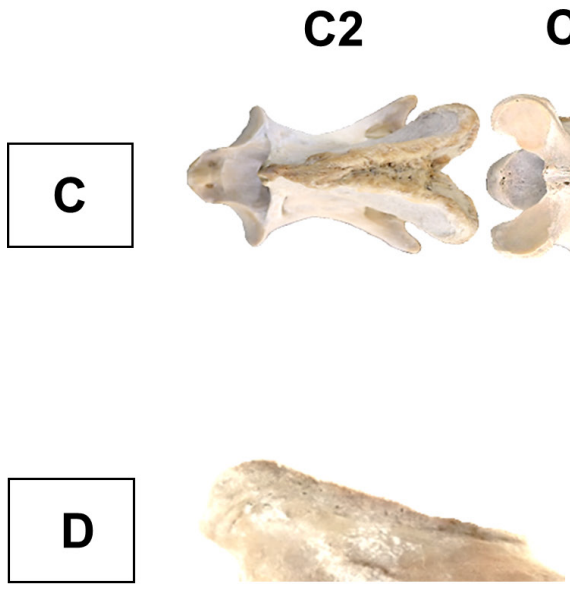
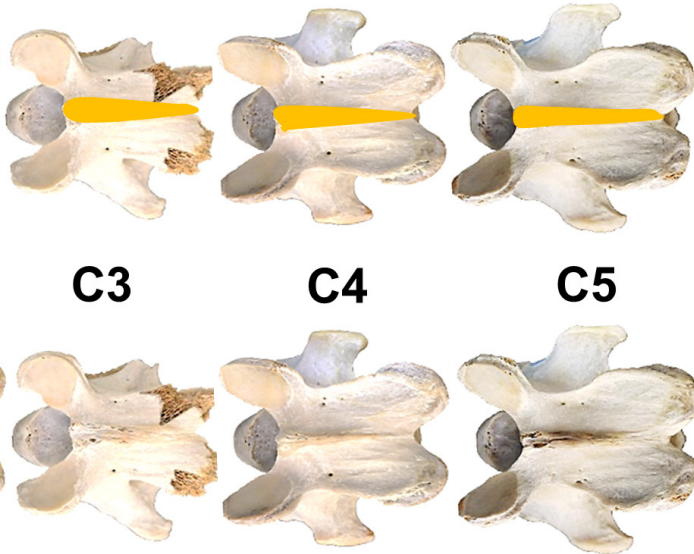

C6
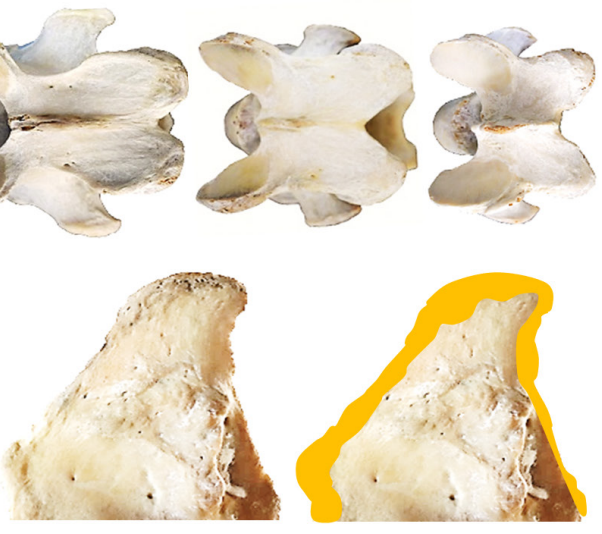

Caudal

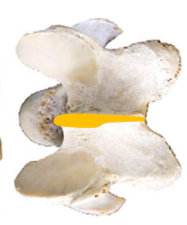

C7
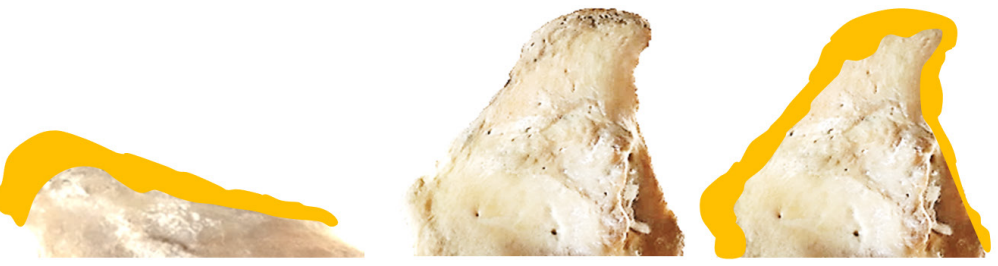

Figure 9. Attachment sites of the ligamentum lamina nuchae in Equus asinus. A, The ligamentum lamina nuchae from the 2 nd - 7th cervical vertebrae. B, dorsal view of the 2 nd -7 th cervical vertebrae showing the mapped attachments sites in orange. C, dorsal view of the entheses patterns. D, lateral view of the dorsal spines of the 6th and 7 th cervical vertebrae showing the mapped attachment sites in orange of the ligamentum lamina nuchae. 
May-Davis et al. - Comparative morphology of ligaments in Equus

2009; Hanot 2017). Therefore, the findings from this study describe new morphological differences between the two clades. This study has been limited by the rarity of some of the species; as a result, it was not possible to perform multiple dissections, and so the effect of intraspecific variation could not be evaluated. Nevertheless, this study identifies a number of potential differences between zebrines and asinines, and provides a baseline for future studies.

In zebrines, the lig. lamina nuchae bears attachments only on the eminences of the cervical dorsal spines in a digitated structure, and due to the specificity of these attachment sites this feature creates a triangular aperture between digitations and correlative enthesis patterns on the dorsal spines. However, when the ligament is absent on the 6th and 7 th cervical vertebrae, the lack of enthesis patterns was also evident. In the asinine, the lig. lamina nuchae forms an uninterrupted attachment to the entire length of the dorsal sagittal ridge in each cervical vertebra, including the dorsal spine of the 7 th cervical vertebra. As a result, the triangular apertures are absent. Furthermore, with a greater number of fibres attaching from the cranial to caudal aspects of the cervical vertebrae, the lig. lamina nuchae creates a longer and wider enthesis pattern in the asinine than zebrines.

In addition, the zebrines possess only the ligg. interspinalia which create a distinct enthesis pattern that clearly differentiates them from the asinine. Although the ligaments are a separate entity in zebrines, the asinine utilizes a morphological variation within the lig. lamina nuchae to apparently accomplish the same function and thus, another discernable variation in enthesis pattern is noted between the two clades. In zebrines, the insertions of the ligg. interspinalia form entheses separate from the lig. lamina nuchae; these entheses are further enhanced when the lig. lamima nuchae is absent. In the asinine, the absence of the ligg. interspinalia provides the lig. lamina nuchae with a continuous and uninterrupted attachment to the dorsal spines. This creates a distinct enthesis pattern that clearly differentiated them from the zebrine.

Consequently, the morphological variations, attachment sites, and presence/absence of the lig. lamina nuchae and ligg. interspinalia between the zebrines and asinine in this study provides a clear musculoskeletal demarcation between the two clades. These findings build on earlier research by May-Davis et al. (2018) that compared the number of attachment sites of the lig. lamina nuchae between in zebrines and asinines. Although the biomechanical relevance is unclear, the factor driving these anatomical variations is likely environmental pressure resulting in evolutionary adaptation. When zebrines diverged from Dinohippus in the late Miocene, they were mixed feeders (grazers and browsers) in an open biome of savannahs, having followed an evolutionary path that combined a more energy efficient form for endurance with increased speed to avoid cursorial carnivores (MacFadden 1992, 2005; Orlando 2015; Figueirido 2015). Thus, biomechanically zebrines developed four locomotive gaits suitable for survival: the walk, trot, canter, and gallop. Of these, the laterally gaited walk, canter, and gallop require head and neck oscillations for biomechanical and energy efficiency (Hoyt 1982; Gellman and Bertram 2002; Clayton 2004; Dunbar et al. 2008).

During locomotion, the dynamic movements of the head and neck have a supportive role over specific gaits, notably the lateral gaits (Hoyt 1981; Clayton 2004). The oscillating movements of the head and neck help to shift the centre of mass and create a torque around the trunk of the zebrine, which in turn generates a greater swing in the distribution of weight between the fore and hindlimbs (Hoyt 1981; Gellman and Bertram 2002; Dunbar et al. 2008; Vincent 2008; Haussler 2016). Subsequently, the lig. lamina nuchae and ligg. interspinalia are of great benefit during these oscillations; being composed of yellow elastin fibres, they function as an energy storage unit during locomotion that helps to supplement the action of muscles while simultaneously preserving energy (Hoyt 1981; Gellman and Bertram 2002; Dunbar et al. 2008; Vincent 2008; Haussler 2016). The lig. lamina nuchae works concurrently with the ligg. interspinalia during locomotion as the former suspends the cervical vertebrae and aids locomotion; while the latter stabilise the cervical vertebrae from the 2 nd cervical vertebra through to the 1 st thoracic vertebra, and together they efficiently promote neck elevation post flexion during oscillating movements. In addition, as the neck provides stability and head positioning, it also supports the vestibular apparatus which helps to hold the visual field in a horizontal plane (Haussler 2016), an essential adaptation for survival in an open environment with pressure from cursorial carnivores. Conversely, asinines evolved to survive in a semiarid, rocky, and mountainous terrain with sparse food sources and intermittent access to water (Dimery 1985; Burnham; Bough 2006; Burden 2015). As a response to its harsh environment, specific adaptations were necessary for survival: small boxy upright hooves for greater accuracy in placement in a harsh browsing environment, specialized skull, mandible and dental morphology to better masticate the fibrous plants rich in lignin, and specific gait adaptations (Dimery 1985; Burnham; Bough 2006; Burden 2015). The preferred gaits for $E$. asinus are walk, amble and trot, which are characterized by minimal oscillations of the head and neck, whereby the amble and trot require the least amount of head/neck movements to support the momentum and equilibrium of the gait (Harris 1993; Clayton 2004; Gonzalez et al. 2018). These three gaits are considered the most energy efficient pathways from both a nutritional and aerobic perspective, especially as speed is not a pressing fac- 
tor in this harsh environment, mainly due to predators facing greater limitations (Hoyt 1981; Dimery 1985; Harris 1993; Burnham 2002; Clayton 2004; Bough 2006; Burden 2015; Figueirido et al. 2015; Gonzalez et al. 2018).

The differing morphology in neck musculature between E. caballus and E. asinus has been well documented, as supported by the findings in this study. For example, the cutaneous colli muscle located in the ventral neck of $E$. asinus is significantly larger and more extensive with greater interconnective properties to surrounding structures than that found in E. caballus (Bough 2006; Herman 2009; Burden 2015). In effect, this provides ventral support in the neck of $E$. asinus during browsing and locomotion while protecting the jugular vein from trauma (Herman 2009). During the biomechanics of amble and trot, the range of motion in the equid's spine is primarily flexion and extension only, which equates to a fixed head and neck position (Harris 1993; Gellman and Bertram 2002; Clayton 2004; Dunbar 2008). With its short neck and proportionally larger head, the head and neck of $E$. asinus requires a different suspension system for locomotion than the longer lever morphology of E. caballus (Clayton 2004; Vincent 2008; Herman 2009). This is one possible explanation for the morphology of the lig. lamina nuchae in relation to the cervical vertebrae and the absence of the ligg. interspinalia in the asinine.

In addition, the extensive attachment sites of the lig. lamina nuchae on the 2nd cervical through to the 1 st thoracic vertebra in E. asinus provides a greater support for suspending the head and neck during its preferred gaits. This passive ligamentous support supplements the action of cervical musculature during locomotion and represents an efficient use of energy (Vincent 2008). Furthermore, as the amble and trot are not lateral gaits, there is minimal to no head/neck oscillation (Gellman and Bertram 2002; Dunbar 2008), thus negating the need for the stabilizing function of the ligg. interspinalia during lateral gaits. This especially applies to a predominantly browsing animal in a fixed body position with ventral neck support from modified musculature (Burnham 2002; Bough 2006; Herman 2009; Burden 2015). This may explain some of the strength and rigidity associated with donkeys in the workplace since domestication, as noted by Hanot et al. (2017).

Understanding equid biomechanics has for the most part been associated with domestic E. caballus, and especially those research projects focused on specific disciplines such as racing, for which specialised breeding programs have been established (Clayton 2004). Since domestication, the horse has undergone a dramatic transformation, having been subjected to intense artificial selection by humans, resulting in over 540 recognised breeds with considerable conformational and biomechanical variations (Rousseau 2014). This intense breeding for specific traits may ex- plain the variations in lig. lamina nuchae attachments to the cervical vertebrae as previously reported in domestic horses (May-Davis and Kleine 2014; May-Davis et al. 2018, 2020). Accordingly, this study may shed light on a wider equid community and offer an opportunity to identify enthesis patterns not yet established in palaeontology, especially between domesticated horses in the zebrine population and the asinine. Therefore, understanding the morphology and attachment sites of the lig. lamina nuchae and ligg. interspinalia to the cervical vertebrae may assist in the identification of cervical vertebral specimens between species of Equus, having now discerned another differentiating osseous morphology between zebrines and asinines.

\section{SUMMARY}

This study describes the morphological variations between the lig. lamina nuchae and ligg. interspinalia and their attachment sites to the cervical vertebrae in four species of Equus. Findings include: differing ligamentous morphology; associative enthesis patterns corresponding to attachment sites of the ligaments to the cervical vertebrae; influences in dorsal spine morphology subject to the presence or absence of the ligaments; and the postulation of evolutionary functional adaptions in response to environmental influences between the two Equus clades. Furthermore, these morphological variations of the cervical vertebrae may provide recognizable features indicative of a species in the identification process for palaeontologists.

\section{ACKNOWLEDGEMENTS}

The authors wish to thank Kristen Brabender, Hortabagy National Park, Hungary, for her assistance with Przewalski horses. Wildlands Zoo for their assistance in a Grant's Zebra, Emmen, The Netherlands; The Australian College of Equine Podiotherapy for the use of its facilities; and the authors/editors/publishers of those articles, journals and books cited in this manuscript.

\section{LITERATURE CITED}

Apostolakos, J., T.J.R. Durant, C.R. Dwyer, R.P. Russell, J.H. Weinreb, F. Alaee, K. Beitzel, M. McCarthy, M.P. Cote, and A.D. Mazzocca. 2014. The enthesis: a review of the tendonto-bone insertion. Muscles, Ligaments and Tendons Journal 4:333-342.

Bendrey, R. 2008. An analysis of factors affecting the development of the equid cranial enthesopathy. Veterinarija Ir Zootechnika 41:26-31.

Benjamin, M., H. Toumi, J.R. Ralphs, G. Bydder, T.M. Best, and S. Milz. 2006. Where tendons and ligaments meet bone: attachment sites ('entheses') in relation to exercise and/or mechanical load. Journal of Anatomy 208:471-490. 
May-Davis et al. - Comparative morphology of ligaments in Equus

Blits, K. 1999. Aristotle: form, function and comparative anatomy. The Anatomical Record 257:58-63.

Bough, J. 2006. From value to vermin: a history of the donkey in Australia. Australian Zoology 33:388-397.

Burden, F. and A. Thiemann. 2015. Donkeys are different. Journal of Equine Veterinary Science 35:376-382.

Burnham, S. 2002. Anatomical differences of the donkey and mule. Proceedings of the Annual Convention of the American Association of Equine Practitioners 48:102-109.

Clayton, H. 2004. The Dynamic Horse. Sport Horse Publications, Mason, USA. 267 pp.

Dimery, N., and R.M. Alexander. 1985. Elastic properties of the hind foot of the donkey, Equus asinus. Journal of Zoology (London) 207:9-20.

Dunbar, D.C., J.M. Macpherson, R.W. Simmons, and A. Zarcades. 2008. Stabilization and mobility moonily of the head, neck and trunk in horses during over-ground locomotion; comparisons with humans and other primates. Journal of Experimental Biology 211:3889-3907.

Dyson, S. 2011. Lesions of the equine neck resulting in lameness or poor performance. Veterinary Clinics of North America: Equine Practice 27:417-437.

Figueirido, B., A. Martin-Serra, Z.J. Tseng, and C.M. Janis. 2015. Habitat changes and changing predatory habits in North American fossil canids. Nature Communication 6:1-11.

Frost, H.M. 1993. Wolff's Law and bone's structural adaptations to mechanical usage: an overview for clinicians. The Angle Orthodontist 64:175-188.

Gasse, H., W. Van Den Broeck. and P. Simeons. 2017. Nomina Anatomica Veterinaria. 6th ed. Published by the Editorial Committee for the World Association of Veterinary Anatomists. Hanover, Ghent, Columbia MO and Rio de Janeiro. 160 pp.

Gellman, K.S. and J.E.A. Bertram. 2002. The equine nuchal ligament 2: passive dynamic energy exchange in locomotion. Veterinary and Comparative Orthopaedics and Traumatology 1:7-14.

González, F.J.N., J.J. Vidal, J.M.L. Jurado, A.K. McLean, G.P. Inostroza, and J.V. Bermejo. 2018. Genetic parameter estimation and implementation of the genetic evaluation for gaits in a breeding program for assisted-therapy in donkeys. Veterinary Research Communication; doi.org/10.1007/S11259-018-9712-6.

Hangey, G. and M. Dingley. 1985. Biological Museum Methods. Academic Press, Sydney Australia. 379 pp.

Hanot, P., A. Herrel, C. Guintard, and R. Cornette. 2017. Morphological integration in the appendicular skeleton of two domestic taxa: the horse and donkey. Proceedings Royal Society 284:20171241.

Harris, S. 1993. Horse Gaits, Balance and Movement. Wiley, New Jersey USA. 178 pp.
Haussler, K.K. June 2016. Functional anatomy and clinical biomechanics of the equine cervical spine. Paper presented at the AAEP Conference $360^{\circ}$ Pain in the Neck, Fort Collins Colorado.

Herman, C.L. 2009. The anatomical differences between the donkey and the horse; in N.S. Matthews and T.S. Taylor (eds), Veterinary Care of Donkeys. International Veterinary Information Service, Ithaca, NY, USA. http://www.ivis.org. (accessed 6th February 2020).

Hoyt, D.F. and C.R. Taylor. 1981. Gait and the energetics of locomotion in horses. Nature 292:239-240.

MacFadden, B.J. 1992. Fossil Horses: Systematics, Paleobiology, and Evolution of the Family Equidae. Cambridge University Press. Cambridge UK. 367 pp.

MacFadden, B.J. 2005. Fossil horses - evidence for evolution. Science 307:1728-1730.

McFadyean, J. 1884. The Anatomy of the Horse; a Dissection Guide. William R. Jenkins. New York USA. 376 pp.

May-Davis, S.E.R. and J. Kleine. 2014. Variations and implications of the gross anatomy in the equine nuchal ligament lamellae. Journal of Equine Veterinary Science 34:1110-1113.

May-Davis, S., W.Y. Brown, and Z. Vermeulen. 2018. The disappearing lamellae: implications of new findings in the family Equidae suggest the loss of nuchal ligament lamellae on C6 and $\mathrm{C} 7$ occurred after domestication. Journal of Equine Veterinary Science 68:108-114.

May-Davis, S., Z. Vermeulen, R. Hunter, W. Brown. 2020. Rare finding of a full nuchal ligament lamellae with attachment points from C2-C7 in one Australian Stock Horse. Journal of Equine Veterinary Science 84:102847.

Orlando, L. 2015. Equids. Current Biology 25, R965-R979.

Rousseau, E. 2014. Horses of the World. Princeton University Press. Princeton UK. 536 pp.

Schlecht, S.H. 2012. Understanding entheses: Bridging the gap between clinical and anthropological perspectives. The Anatomical Record 295:1239-1251.

Shaw, H.M. and M. Benjamin. 2007. Structure-function relationships of entheses in relation to mechanical load and exercise. Scandinavian Journal of Medicine \& Science in Sports 17:303-315.

Sisson, S. 1975. Equine syndesmology. The anatomy of domestic animals; pp. 349-375 in S. Sisson, and J.D. Grossman (eds.), The Anatomy of the Domestic Animals. Saunders. Philadelphia USA.

Vilstrup, J.T., A. Seguin-Orlando, M. Stiller, A. Ginolhac, M. Raghavan, S.C.A. Nielsen, J. Weinstock, D. Froese, S.K. Vasiliev, N.D. Ovodov, J. Clary, K.M. Helgen, R.C. Fleischer, A. Cooper, B. Shapiro, and L. Orlando. 2013. Mitochondrial phylogenomics of modern and ancient equids. PLOS one 8: e55950.

Vincent, J.F.R. 2008. Applications - some influences of engineering ideas in biology. Journal of Biological Engineering 3:99-114. 